\title{
Some new finite difference inequalities arising in the theory of difference equations
}

Qinghua Feng ${ }^{1,2^{*}}$, Fanwei Meng ${ }^{2}$ and Yaoming Zhang ${ }^{1}$

* Correspondence: fqhua@sina.com 'school of Science, Shandong University of Technology, Zhangzhou Road 12, Zibo, Shandong, 255049, China

Full list of author information is available at the end of the article

\section{Abstract}

In this work, some new finite difference inequalities in two independent variables are established, which can be used in the study of qualitative as well as quantitative properties of solutions of certain difference equations. The established results extend some existing results in the literature.

MSC 2010: 26D15

Keywords: Finite difference inequalities, Difference equations, Explicit bounds, Qualitative analysis, Quantitative analysis

\section{Introduction}

Finite difference inequalities in one or two independent variables which provide explicit bounds play a fundamental role in the study of boundedness, uniqueness, and continuous dependence on initial data of solutions of difference equations. Many difference inequalities have been established (for example, see [1-11] and the references therein). In the research of difference inequalities, generalization of known inequalities has been paid much attention by many authors. Here we list some recent results in the literature.

In [[12], Theorems 2.6-2.8], Pachpatte presents the following six discrete inequalities, based on which some new bounds on unknown functions are established.

$$
\left(a_{1}\right): u(m, n) \leq a(m, n)+b(m, n) \sum_{s=0}^{m-1} \sum_{t=n+1}^{\infty} c(s, t) u(s, t)
$$

Preprint submitted to Advances in Difference Equations June 16, 2011

$$
\begin{aligned}
& \left(a_{2}\right): u(m, n) \leq a(m, n)+b(m, n) \sum_{s=m+1}^{\infty} \sum_{t=n+1}^{\infty} c(s, t) u(s, t), \\
& \left(a_{3}\right): u(m, n) \leq a(m, n)+\sum_{s=0}^{m-1} b(s, n) u(s, n)+\sum_{s=m+1}^{\infty} \sum_{t=n+1}^{\infty} c(s, t) u(s, t), \\
& \left(a_{4}\right): u(m, n) \leq a(m, n)+\sum_{s=m+1}^{\infty} b(s, n) u(s, n)+\sum_{s=m+1}^{\infty} \sum_{t=n+1}^{\infty} c(s, t) u(s, t),
\end{aligned}
$$

\section{SpringerOpen ${ }^{\circ}$}

(c) 2011 Feng et al; licensee Springer. This is an Open Access article distributed under the terms of the Creative Commons Attribution License (http://creativecommons.org/licenses/by/2.0), which permits unrestricted use, distribution, and reproduction in any medium, provided the original work is properly cited. 


$$
\begin{aligned}
& \left(a_{5}\right): u(m, n) \leq a(m, n)+\sum_{s=0}^{m-1} b(s, n) u(s, n)+\sum_{s=0}^{m-1} \sum_{t=n+1}^{\infty} L(s, t, u(s, t)) \\
& \left(a_{6}\right): u(m, n) \leq a(m, n)+\sum_{s=m+1}^{\infty} b(s, n) u(s, n)+\sum_{s=m+1}^{\infty} \sum_{t=n+1}^{\infty} L(s, t, u(s, t))
\end{aligned}
$$

where $u, a, b, c$ are nonnegative functions defined on $m \in \mathbb{N}_{0}, n \in \mathbb{N}_{0}$, and $L: \mathbb{N}_{0} \times$ $\mathbb{N}_{0} \times \mathbb{R}_{+} \rightarrow \mathbb{R}_{+}$satisfies $0 \leq L(m, n, u)-L(m, n, v) \leq M(m, n, v)(u-v)$ for $u \geq v \geq 0$, where $M: \mathbb{N}_{0} \times \mathbb{N}_{0} \times \mathbb{R}_{+} \rightarrow \mathbb{R}_{+}$.

Recently, in [[13], Theorems 1-6], Meng and Li present the following inequalities with more general forms.

$$
\begin{aligned}
& (b 1): u^{p}(m, n) \leq a(m, n)+b(m, n) \sum_{s=0}^{m-1} \sum_{t=n+1}^{\infty}[c(s, t) u(s, t)+e(s, t)], \\
& (b 2): u^{p}(m, n) \leq a(m, n)+b(m, n) \sum_{s=m+1}^{\infty} \sum_{t=n+1}^{\infty}[c(s, t) u(s, t)+e(s, t)], \\
& (b 3): u^{p}(m, n) \leq a(m, n)+\sum_{s=0}^{m-1} b(s, n) u^{p}(s, n)+\sum_{s=0}^{m-1} \sum_{t=n+1}^{\infty}[c(s, t) u(s, t)+e(s, t)], \\
& (b 4): u^{p}(m, n) \leq a(m, n)+\sum_{s=m+1}^{\infty} b(s, n) u^{p}(s, n)+\sum_{s=m+1}^{\infty} \sum_{t=n+1}^{\infty}[c(s, t) u(s, t)+e(s, t)] . \\
& \left(b_{5}\right): u^{p}(m, n) \leq a(m, n)+\sum_{s=0}^{m-1} b(s, n) u^{p}(s, n)+\sum_{s=0}^{m-1} \sum_{t=n+1}^{\infty} L(s, t, u(s, t)), \\
& \left(b_{6}\right): u^{p}(m, n) \leq a(m, n)+\sum_{s=m+1}^{\infty} b(s, n) u^{p}(s, n)+\sum_{s=m+1}^{\infty} \sum_{t=n+1}^{\infty} L(s, t, u(s, t)),
\end{aligned}
$$

where $p \geq 1$ is a constant, $u, a, b, c, e$ are nonnegative functions defined on $m \in \mathbb{N}_{0}$, $n \in \mathbb{N}_{0}$, and $L$ is defined the same as in (a5)-a(6).

As one can see, $(b 1)-(b 2)$ are generalizations of (a1)-(a2), while (b4)-(b6) are generalizations of (a4)-(a6).

More recently, Meng and Ji [[14], Theorems 3, 4, 7, 8] extended (b1)-(b4) to the following inequalities.

$$
\begin{aligned}
& (c 1): u^{p}(m, n) \leq a(m, n)+b(m, n) \sum_{s=0}^{m-1} \sum_{t=n+1}^{\infty}\left[c(s, t) u^{q}(s, t)+d(s, t) u^{r}(s, t)+e(s, t)\right], \\
& \left.(c 2): u^{p}(m, n) \leq a(m, n)+b(m, n) \sum_{s=m+1}^{\infty} \sum_{t=n+1}^{\infty}\left[c(s, t) u^{q}(s, t)+d(s, t) u^{r}(s, t)+e(s, t)\right]\right] \\
& (c 3): u^{p}(m, n) \leq a(m, n)+\sum_{s=0}^{m-1} b(s, n) u^{p}(s, n)+\sum_{s=0}^{m-1} \sum_{t=n+1}^{\infty}\left[c(s, t) u^{q}(s, t)+d(s, t) u^{r}(s, t)+e(s, t)\right]
\end{aligned}
$$




$$
(c 4): u^{p}(m, n) \leq a(m, n)+\sum_{s=m+1}^{\infty} b(s, n) u^{p}(s, n)+\sum_{s=m+1}^{\infty} \sum_{t=n+1}^{\infty}\left[c(s, t) u^{q}(s, t)+d(s, t) u^{r}(s, t)+e(s, t)\right] .
$$

where $p, q, r$ are constants with $p \geq q, p \geq r, p \neq 0$, and $u, a, b, c, d, e$ are nonnegative functions defined on $m \in \mathbb{N}_{0}, n \in \mathbb{N}_{0}$.

The presented inequalities above have proved to be very useful in the study of quantitative as well as qualitative properties of solutions of certain difference equations.

Motivated by the work mentioned above, in this paper, we will establish some more generalized finite difference inequalities, which provide new bounds for unknown functions lying in these inequalities. We will illustrate the usefulness of the established results by applying them to study the boundedness, uniqueness, and continuous dependence on initial data of solutions of certain difference equations.

Throughout this paper, $\mathbb{R}$ denotes the set of real numbers and $\mathbb{R}_{+}=[0, \infty)$, and $\mathbb{Z}$ denotes the set of integers, while $\mathbb{N}_{0}$ denotes the set of nonnegative integers. $I:=\left[m_{0}, \infty\right] \cap$ $\mathbb{Z}$ and $\widehat{I}:=\left[n_{0}, \infty\right] \bigcap \mathbb{Z}$ are two fixed lattices of integral points in $\mathbb{R}$, where $m_{0}, n_{0} \in \mathbb{Z}$. Let $\Omega:=I \times \widehat{I} \subset \mathbb{Z}^{2}$. We denote the set of all $\mathbb{R}$-valued functions on $\Omega$ by $\wp(\Omega)$, and denote the set of all $\mathbb{R}_{+}$-valued functions on $\Omega$ by $\wp_{+}(\Omega)$. The partial difference operators $\Delta_{1}$ and $\Delta_{2}$ on $u \in \wp(\Omega)$ are defined as $\Delta_{1} u(m, n)=u(m+1, n)-u(m, n), \Delta_{2} u(m, n)=u$ $(m, n+1)-u(m, n)$.

\section{Main results}

Lemma 2.1. [[15]] Assume that $a \geq 0, p \geq q \geq 0$, and $p \neq 0$, then for any $K>0$

$$
a^{\frac{q}{p}} \leq \frac{q}{p} K^{\frac{q-p}{p}} a+\frac{p-q}{p} K^{\frac{q}{p}}
$$

Lemma 2.2. Let $u(m, n), a(m, n), b(m, n)$ are nonnegative functions defined on $\Omega$ with $a(m, n)$ not equivalent to zero.

(1) Assume that $a(m, n)$ is nondecreasing in the first variable. If

$$
u(m, n) \leq a(m, n)+\sum_{s=m_{0}}^{m-1} b(s, n) u(s, n)
$$

for $(m, n) \in \Omega$, then

$$
u(m, n) \leq a(m, n) \prod_{s=m_{0}}^{m-1}[1+b(s, n)]
$$

(2) Assume that $a(m, n)$ is decreasing in the first variable. If

$$
u(m, n) \leq a(m, n)+\sum_{s=m+1}^{\infty} b(s, n) u(s, n)
$$

for $(m, n) \in \Omega$, then

$$
u(m, n) \leq a(m, n) \prod_{s=m+1}^{\infty}[1+b(s, n)] .
$$


Remark 1. Lemma 2.2 is a direct variation of [[12], Lemma 2.5].

Theorem 2.1. Suppose $u, a, b, f, g, h, w \in \wp_{+}(\Omega)$, and $b, f, g, h, w$ are nondecreasing in the first variable, while decreasing in the second variable. $\alpha: I \rightarrow I$ is nondecreasing with $\alpha(m) \leq m$ for $\forall m \in I$, while $\beta: \widehat{I} \rightarrow \widehat{I}$ is nondecreasing with $\beta(n) \geq n$ for $\forall n \in \widehat{I}$. $p, q, r, l$ are constants with $p \geq q, p \geq r, p \geq l, p \neq 0$.

If for $(m, n) \in \Omega, u(m, n)$ satisfies the following inequality

$$
u^{p}(m, n) \leq a(m, n)+b(m, n) \sum_{s=\alpha\left(m_{0}\right)}^{\alpha(m)-1} \sum_{t=\beta(n)+1}^{\infty}\left[f(s, t) u^{q}(s, t)+g(s, t) u^{r}(s, t)+h(s, t)+\sum_{\xi=0}^{s} \sum_{\eta=t}^{\infty} w(\xi, \eta) u^{l}(\xi, \eta)\right]
$$

then we have

$$
\begin{gathered}
u(m, n) \leq\left\{a(m, n)+b(m, n) H(m, n) \prod_{s=m_{0}}^{m-1}\left\{1+\sum_{t=n+1}^{\infty}[\alpha(s+1)-\alpha(s)][\beta(t)-\beta(t-1)]\right.\right. \\
\left.\left.\left[\bar{f}(s, t) \frac{q}{p} K^{\frac{q-p}{p}}+\bar{g}(s, t) \frac{r}{p} K^{\frac{r-p}{p}}+\sum_{\xi=0}^{s} \sum_{\eta=t}^{\infty} \bar{w}(\xi, \eta) \frac{l}{p} K^{\frac{l-p}{p}}\right]\right\}\right\}^{\frac{1}{p}}
\end{gathered}
$$

provided $H(m . n)>0$, where $K>0$ is a constant, and

$$
\left\{\begin{array}{c}
H(m, n)=\sum_{s=\alpha\left(m_{0}\right)}^{\alpha(m)-\beta(n)+1} \sum^{\infty}\left\{f(s, t)\left[\frac{q}{p} K^{\frac{q-p}{p}} a(s, t)+\frac{p-q}{p} K^{\frac{q}{p}}\right]+g(s, t)\left[\frac{r}{p} K^{\frac{r-p}{p}} a(s, t)+\frac{p-r}{p} K^{\frac{r}{p}}\right]\right. \\
\left.+h(s, t)+\sum_{\xi=0}^{s} \sum_{\eta=t}^{\infty} w(\xi, \eta)\left[\frac{l}{p} K^{\frac{l-p}{p}} a(\xi, \eta)+\frac{p-l}{p} K^{\frac{l}{p}}\right]\right\}, \\
\bar{f}=f(m, n) b(m, n), \bar{g}=g(m, n) b(m, n), \bar{w}=w(m, n) b(m, n) .
\end{array}\right.
$$

Proof. Let $z(m, n)=\sum_{s=\alpha\left(m_{0}\right)}^{\alpha(m)-1} \sum_{t(n)+1}^{\infty}\left[f(s, t) u^{q}(s, t)+g(s, t) u^{r}(s, t)+h(s, t)+\sum_{\xi=0}^{s} \sum_{\eta=t}^{\infty} f(\xi, \eta) u^{l}(\xi, \eta)\right]$.

Then we have

$$
u(m, n) \leq[a(m, n)+b(m, n) z(m, n)]^{\frac{1}{p}} .
$$

Furthermore, if given $(X, Y) \in \Omega$, and $(m, n) \in\left(\left[m_{0}, X\right] \times[Y, \infty]\right) \cap \Omega$, then using (4) and Lemma 2.1 we have

$$
\begin{aligned}
z(m, n) \leq & \sum_{s=\alpha\left(m_{0}\right)}^{\alpha(m)-1} \sum_{t=\beta(n)+1}^{\infty}\left\{f(s, t)[a(s, t)+b(s, t) z(s, t)]^{\frac{q}{p}}+g(s, t)[a(s, t)+b(s, t) z(s, t)]^{\frac{r}{p}}\right. \\
& \left.+h(s, t)+\sum_{\xi=0}^{s} \sum_{\eta=t}^{\infty} w(\xi, \eta)[a(\xi, \eta)+b(\xi, \eta) z(\xi, \eta)]^{\frac{l}{p}}\right\} \\
\leq & \sum_{s=\alpha\left(m_{0}\right)}^{\alpha(m)-1} \sum_{t=\beta(n)+1}^{\infty}\left\{f(s, t)\left[\frac{q}{p} K^{\frac{q-p}{p}}(a(s, t)+b(s, t) z(s, t))+\frac{p-q}{p} K^{\frac{q}{p}}\right]\right. \\
& +g(s, t)\left[\frac{r}{p} K^{\frac{r-p}{p}}(a(s, t)+b(s, t) z(s, t))+\frac{p-r}{p} K^{\frac{r}{p}}\right] \\
& \left.+h(s, t)+\sum_{\xi=0}^{s} \sum_{\eta=t}^{\infty} w(\xi, \eta)\left[\frac{l}{p} K^{\frac{l-p}{p}}(a(\xi, \eta)+b(\xi, \eta) z(\xi, \eta))+\frac{p-l}{p} K^{\frac{l}{p}}\right]\right\} \\
= & H(m, n)+\sum_{s=\alpha\left(m_{0}\right)}^{\alpha(m)-1} \sum_{=\beta(n)+1}^{\infty}\left\{f(s, t) b(s, t) \frac{q}{p} K^{\frac{q-p}{p}} z(s, t)+g(s, t) b(s, t) \frac{r}{p} K^{\frac{r-p}{p}} z(s, t)\right. \\
& \left.+\sum_{\xi=0}^{s} \sum_{\eta=t}^{\infty} w(\xi, \eta) b(\xi, \eta) \frac{l}{p} K^{\frac{l-p}{p}} z(\xi, \eta)\right\} \\
\leq & H(X, Y)+\sum_{\left.s=\alpha\left(m_{0}\right)\right)}^{\alpha(m)-1} \sum_{t=\beta(n)+1}^{\infty} \bar{f}(s, t) \frac{q}{p} K^{\frac{q-p}{p}} z(s, t)+\bar{g}(s, t) \frac{r}{p} K^{\frac{r-p}{p}} z(s, t) \\
& \left.+\sum_{\xi=0}^{s} \sum_{\eta=t}^{\infty} \bar{w}(\xi, \eta) \frac{l}{p} K^{\frac{l-p}{p}} z(\xi, \eta)\right\},
\end{aligned}
$$


where $H, \bar{f}, \bar{g}, \bar{w}$ are defined in (3).

Let the right side of $(5)$ be $v(m, n)$. Then

$$
z(m, n) \leq v(m, n),
$$

and

$$
\begin{aligned}
& {[v(m+1, n)-v(m, n)]-[v(m+1, n+1)-v(m, n+1)]} \\
& =\sum_{s=\alpha(m)}^{\alpha(m+1)-1} \sum_{t=\beta(n)+1}^{\beta(n+1)}\left[\bar{f}(s, t) \frac{q}{p} K^{\frac{q-p}{p}} z(s, t)+\bar{g}(s, t) \frac{r}{p} K^{\frac{r-p}{p}} z(s, t)+\sum_{\xi=0}^{s} \sum_{\eta=t}^{\infty} \bar{w}(\xi, \eta) \frac{l}{p} K^{\frac{l-p}{p}} z(\xi, \eta)\right] \\
& \leq \sum_{s=\alpha(m)}^{\alpha(m+1)-1} \sum_{t=\beta(n)+1}^{\beta(n+1)}\left[\bar{f}(s, t) \frac{q}{p} K^{\frac{q-p}{p}} v(s, t)+\bar{g}(s, t) \frac{r}{p} K^{\frac{r-p}{p}} v(s, t)+\sum_{\xi=0}^{s} \sum_{\eta=t}^{\infty} \bar{w}(\xi, \eta) \frac{l}{p} K^{\frac{l-p}{p}} v(\xi, \eta)\right] \\
& \leq \sum_{s=\alpha(m)}^{\alpha(m+1)-1} \sum_{t=\beta(n)+1}^{\beta(n+1)}\left[\bar{f}(s, t) \frac{q}{p} K^{\frac{q-p}{p}}+\bar{g}(s, t) \frac{r}{p} K^{\frac{r-p}{p}}+\sum_{\xi=0}^{s} \sum_{\eta=t}^{\infty} \bar{w}(\xi, \eta) \frac{l}{p} K^{\frac{l-p}{p}}\right] v(s, t) \\
& \leq[\alpha(m+1)-\alpha(m)][\beta(n+1)-\beta(n)]\left[\bar{f}(\alpha(m+1)-1, \beta(n)+1) \frac{q}{p} K^{\frac{q-p}{p}}\right. \\
& \left.+\bar{g}(\alpha(m+1)-1, \beta(n)+1) \frac{r}{p} K^{\frac{r-p}{p}}+\sum_{\xi=0}^{\alpha(m+1)-1} \sum_{\eta=\beta(n)+1}^{\infty} \bar{w}(\xi, \eta) \frac{l}{p} K^{\frac{l-p}{p}}\right] v(\alpha(m+1)-1, \beta(n)+1) \\
& \leq[\alpha(m+1)-\alpha(m)][\beta(n+1)-\beta(n)]\left[\bar{f}(m, n+1) \frac{q}{p} K^{\frac{q-p}{p}}+\bar{g}(m, n+1) \frac{r}{p} K \frac{r-p}{p}\right. \\
& \left.+\sum_{\xi=0}^{m} \sum_{\eta=n+1}^{\infty} \bar{w}(\xi, \eta) \frac{l}{p} K^{\frac{l-p}{p}}\right] v(m, n+1) .
\end{aligned}
$$

Considering $v(m, n) \geq v(m, n+1)$, we have

$$
\begin{aligned}
& \frac{v(m+1, n)-v(m, n)}{v(m, n)}-\frac{v(m+1, n+1)-v(m, n+1)}{v(m, n+1)} \\
& \leq[\alpha(m+1)-\alpha(m)][\beta(n+1)-\beta(n)]\left[\bar{f}(m, n+1) \frac{q}{p} K^{\frac{q-p}{p}}+\bar{g}(m, n+1) \frac{r}{p} K^{\frac{r-p}{p}}+\sum_{\xi=0}^{m} \sum_{\eta=n+1}^{\infty} \bar{w}(\xi, \eta) \frac{l}{p} K^{\frac{l-p}{p}}\right] .
\end{aligned}
$$

Setting $n=t$ in (7), and a summary with respect to $t$ from $n$ to $r-1$ yields

$$
\begin{aligned}
& \frac{v(m+1, n)-v(m, n)}{v(m, n)}-\frac{v(m+1, r)-v(m, r)}{v(m, r)} \\
& \quad \leq \sum_{t=n+1}^{r}[\alpha(m+1)-\alpha(m)][\beta(t)-\beta(t-1)]\left[\bar{f}(m, t) \frac{q}{p} K^{\frac{q-p}{p}}+\bar{g}(m, t) \frac{r}{p} K^{\frac{r-p}{p}}+\sum_{\xi=0}^{m} \sum_{\eta=t}^{\infty} \bar{w}(\xi, \eta) \frac{l}{p} K^{\frac{l-p}{p}}\right] .
\end{aligned}
$$

Letting $r \rightarrow \infty$ in (8), using $v(m, \infty)=H(X, Y)$ we obtain

$$
\begin{aligned}
& \frac{v(m+1, n)-v(m, n)}{v(m, n)} \\
& \leq \sum_{t=n+1}^{\infty}[\alpha(m+1)-\alpha(m)][\beta(t)-\beta(t-1)]\left[\bar{f}(m, t) \frac{q}{p} K^{\frac{q-p}{p}}+\bar{g}(m, t) \frac{r}{p} K^{\frac{r-p}{p}}+\sum_{\xi=0}^{m} \sum_{\eta=t}^{\infty} \bar{w}(\xi, \eta) \frac{l}{p} K^{\frac{l-p}{p}}\right],
\end{aligned}
$$

which is followed by

$$
\frac{v(m+1, n)}{v(m, n)} \leq\left\{1+\sum_{t=n+1}^{\infty}[\alpha(m+1)-\alpha(m)][\beta(t)-\beta(t-1)]\left[\bar{f}(m, t) \frac{q}{p} K^{\frac{q-p}{p}}+\bar{g}(m, t) \frac{r}{p} K^{\frac{r-p}{p}}+\sum_{\xi=0}^{m} \sum_{n=t}^{\infty} \bar{w}(\xi, n) \frac{l}{p} K^{\frac{l-p}{p}}\right]\right\}
$$

Setting $m=s$ in (9), and a multiple with respect to $s$ from $m_{0}$ to $m$ - 1 yields

$$
\frac{v(m, n)}{v\left(m_{0}, n\right)} \leq \prod_{s=m_{0}}^{m-1}\left\{1+\sum_{t=n+1}^{\infty}[\alpha(s+1)-\alpha(s)][\beta(t)-\beta(t-1)]\left[\bar{f}(s, t) \frac{q}{p} K^{\frac{q-p}{p}}+\bar{g}(s, t) \frac{r}{p} K^{\frac{r-p}{p}}+\sum_{\xi=0}^{s} \sum_{\eta=t}^{\infty} w(\xi, \eta) \frac{l}{p} K^{\frac{l-p}{p}}\right]\right\} .
$$


Considering $v\left(m_{0}, n\right)=H(X, Y)$, and then combining (4), (6) and (10) we obtain

$$
\begin{gathered}
u(m, n) \leq\left\{a(m, n)+b(m, n) H(X, Y) \prod_{s=m_{0}}^{m-1}\left\{1+\sum_{t=n+1}^{\infty}[\alpha(s+1)-\alpha(s)][\beta(t)-\beta(t-1)]\right.\right. \\
\left.\left.\left[\bar{f}(s, t) \frac{q}{p} K^{\frac{q-p}{p}}+\bar{g}(s, t) \frac{r}{p} K^{\frac{r-p}{p}}+\sum_{\xi=0}^{s} \sum_{\eta=t}^{\infty} \bar{w}(\xi, \eta) \frac{l}{p} K^{\frac{l-p}{p}}\right]\right\}\right\}^{\frac{1}{p}} .
\end{gathered}
$$

Setting $m=X, n=Y$ in (11), and considering $(X, Y) \in \Omega$ is selected arbitrarily, then after substituting $X, Y$ with $m, n$ we obtain the desired inequality.

Remark 2. If we take $\Omega=\mathbb{N}_{0} \times \mathbb{N}_{0}, w(m, n) \equiv 0, \alpha(m)=m, \beta(n)=n$, and omit the conditions " $b, f, g, h, w$ are nondecreasing in the first variable, while decreasing in the second variable" in Theorem 2.1, which is unnecessary for the proof since $\alpha(m)=m, \beta$ $(n)=n$, then Theorem 2.1 reduces to [[14], Theorem 3]. Furthermore, if $g(m, n) \equiv 0$, $q=1, p \geq 1$, then Theorem 2.1 reduces to [[13], Theorem 1].

Following a similar process as the proof of Theorem 2.1, we have the following three theorems.

Theorem 2.2. Suppose $u, a, b, f, g, h, w \in \wp_{+}(\Omega)$, and $b, f, g, h, w$ are decreasing both in the first variable and the second variable. $\alpha: I \rightarrow I$ is nondecreasing with $\alpha(m)$ $\geq m$ for $\forall m \in I$, while $\beta: \widehat{I} \rightarrow \widehat{I}$ is nondecreasing with $\beta(n) \geq n$ for $\forall n \in \widehat{I} \cdot p, q, r, l$ are defined as in Theorem 2.1. If for $(m, n) \in \Omega, u(m, n)$ satisfies the following inequality

$$
u^{p}(m, n) \leq a(m, n)+b(m, n) \sum_{s=\alpha(m)+1}^{\infty} \sum_{t=\beta(n)+1}^{\infty}\left[f(s, t) u^{q}(s, t)+g(s, t) u^{r}(s, t)+h(s, t)+\sum_{\xi=s}^{\infty} \sum_{\eta=t}^{\infty} w(\xi, \eta) u^{l}(\xi, \eta)\right],
$$

then we have

$$
\begin{gathered}
u(m, n) \leq\left\{a(m, n)+b(m, n) H(m, n) \prod_{s=\alpha(m)+1}^{\infty}\left\{1+\sum_{t=n+1}^{\infty}[\alpha(s+1)-\alpha(s)][\beta(t)-\beta(t-1)]\right.\right. \\
\left.\left.\left[\bar{f}(s, t) \frac{q}{p} K^{\frac{q-p}{p}}+\bar{g}(s, t) \frac{r}{p} K^{\frac{r-p}{p}}+\sum_{\xi=s}^{\infty} \sum_{\eta=t}^{\infty} \bar{w}(\xi, \eta) \frac{l}{p} K^{\frac{l-p}{p}}\right]\right\}\right\}^{\frac{1}{p}}
\end{gathered}
$$

provided $H(m . n)>0$, where $\bar{f}, \bar{g}, \bar{w}$ are defined as in Theorem 2.1, and

$$
\begin{gathered}
H(m, n)=\sum_{s=\alpha(m)+1}^{\infty} \sum_{t=\beta(n)+1}^{\infty}\left\{f(s, t)\left[\frac{q}{p} K^{\frac{q-p}{p}} a(s, t)+\frac{p-q}{p} K^{\frac{q}{p}}\right]+g(s, t)\left[\frac{r}{p} K^{\frac{r-p}{p}} a(s, t)+\frac{p-r}{p} K^{\frac{r}{p}}\right]\right. \\
\left.+h(s, t)+\sum_{\xi=s}^{\infty} \sum_{\eta=t}^{\infty} w(\xi, \eta)\left[\frac{l}{p} K^{\frac{l-p}{p}} a(\xi, \eta)+\frac{p-l}{p} K^{\frac{l}{p}}\right]\right\} .
\end{gathered}
$$

Remark 3. If we take $\Omega=\mathbb{N}_{0} \times \mathbb{N}_{0}, w(m, n) \equiv 0, \alpha(m)=m, \beta(n)=n$, and omit the conditions " $b, f, g, h, w$ are decreasing both in the first variable and the second variable" in Theorem 2.2, which are unnecessary for the proof since $\alpha(m)=m, \beta(n)=n$, then Theorem 2.2 reduces to [[14], Theorem 4]. Furthermore, if $g(m, n) \equiv 0, q=1, p \geq$ 1, then Theorem 2.2 reduces to [[13], Theorem 2].

Theorem 2.3. Suppose $u, a, b, f, g, h, w \in \wp_{+}(\Omega)$, and $b, f, g, h, w$ are nondecreasing both in the first variable and the second variable. $\alpha: I \rightarrow I$ is nondecreasing with $\alpha(m)$ $\leq m$ for $\forall m \in I$, while $\beta: \widehat{I} \rightarrow \widehat{I}$ is nondecreasing with $\beta(n) \leq n$ for $\forall n \in \widehat{I} \cdot p, q, r, l$ are defined as in Theorem 2.1. If for $(m, n) \in \Omega, u(m, n)$ satisfies the following inequality 


$$
\left.u^{p}(m, n) \leq a(m, n)+b(m, n) \sum_{s=\alpha\left(m_{0}\right) t=\beta\left(n_{0}\right)}^{\alpha(m)-1} \sum_{\beta(n)-1} f(s, t) u^{q}(s, t)+g(s, t) u^{r}(s, t)+h(s, t)+\sum_{\xi=0}^{s} \sum_{\eta=0}^{t} w(\xi, \eta) u^{l}(\xi, \eta)\right]
$$

then we have

$$
\begin{gathered}
u(m, n) \leq\left\{a(m, n)+b(m, n) H(m, n) \prod_{s=m_{0}}^{m-1}\left\{1+\sum_{t=n_{0}}^{n-1}[\alpha(s+1)-\alpha(s)][\beta(t)-\beta(t-1)]\right.\right. \\
\left.\left.\left[f(s, t) \frac{q}{p} K^{\frac{q-p}{p}}+g(s, t) \frac{r}{p} K^{\frac{r-p}{p}}+\sum_{\xi=0}^{s} \sum_{\eta=0}^{t} w(\xi, \eta) \frac{l}{p} K^{\frac{l-p}{p}}\right]\right\}\right\}
\end{gathered}
$$

provided $H(m . n)>0$, where $\bar{f}, \bar{g}, \bar{w}$ are defined as in Theorem 2.1 , and

$$
\begin{aligned}
H(m, n)= & \sum_{s=\alpha\left(m_{0}\right)}^{\alpha(m)-1} \sum_{t=\beta\left(n_{0}\right)}^{\beta(n)-1}\left\{f(s, t)\left[\frac{q}{p} K \frac{q-p}{p} a(s, t)+\frac{p-q}{p} K \frac{q}{p}\right]+g(s, t)\left[\frac{r}{p} K \frac{r-p}{p} a(s, t)+\frac{p-r}{p} K \frac{r}{p}\right]\right. \\
& \left.+h(s, t)+\sum_{\xi=0}^{s} \sum_{\eta=0}^{t} w(\xi, \eta)\left[\frac{l}{p} K^{\frac{l-p}{p}} a(\xi, \eta)+\frac{p-l}{p} K^{\frac{l}{p}}\right]\right\} .
\end{aligned}
$$

Theorem 2.4. Suppose $u, a, b, f, g, h, w \in \wp_{+}(\Omega)$, and $b, f, g, h, w$ are decreasing in the first variable, while nondecreasing in the second variable. $\alpha: I \rightarrow I$ is nondecreasing with $\alpha(m) \geq m$ for $\forall m \in I$, while $\beta: \widehat{I} \rightarrow \widehat{I}$ is nondecreasing with $\beta(n) \leq n$ for $\forall n \in \widehat{I} \cdot p, q, r, l$ are defined as in Theorem 2.1. If for $(m, n) \in \Omega, u(m, n)$ satisfies the following inequality

$$
u^{p}(m, n) \leq a(m, n)+b(m, n) \sum_{s=\alpha(m)+1}^{\infty} \sum_{t=\beta\left(n_{0}\right)}^{\beta(n)-1}\left[f(s, t) u^{q}(s, t)+g(s, t) u^{r}(s, t)+h(s, t)+\sum_{\xi=s}^{\infty} \sum_{\eta=0}^{t} w(\xi, \eta) u^{l}(\xi, \eta)\right]
$$

then we have

$$
\begin{gathered}
u(m, n) \leq\left\{a(m, n)+b(m, n) H(m, n) \prod_{s=\alpha(m)+1}^{\infty}\left\{1+\sum_{t=n_{0}}^{n-1}[\alpha(s+1)-\alpha(s)][\beta(t)-\beta(t-1)]\right.\right. \\
\left.\left.\left[f(s, t) \frac{q}{p} K^{\frac{q-p}{p}}+g(s, t) \frac{r}{p} K^{\frac{r-p}{p}}+\sum_{\xi=s}^{\infty} \sum_{\eta=0}^{t} w(\xi, \eta) \frac{l}{p} K^{\frac{l-p}{p}}\right]\right\}\right\}^{\frac{1}{p}}
\end{gathered}
$$

provided $H(m . n)>0$, where $\bar{f}, \bar{g}, \bar{w}$ are defined as in Theorem 2.1 , and

$$
\begin{aligned}
H(m, n)= & \sum_{s=\alpha(m)+1}^{\infty} \sum_{t=\beta\left(n_{0}\right)}^{\beta(n)-1}\left\{f(s, t)\left[\frac{q}{p} K^{\frac{q-p}{p}} a(s, t)+\frac{p-q}{p} K^{\frac{q}{p}}\right]+g(s, t)\left[\frac{r}{p} K^{\frac{r-p}{p}} a(s, t)+\frac{p-r}{p} K^{\frac{r}{p}}\right]\right. \\
& \left.+h(s, t)+\sum_{\xi=s}^{\infty} \sum_{\eta=0}^{t} w(\xi, \eta)\left[\frac{l}{p} K^{\frac{l-p}{p}} a(\xi, \eta)+\frac{p-l}{p} K^{\frac{l}{p}}\right]\right\} .
\end{aligned}
$$

Next we will study the following difference inequality:

$$
\begin{aligned}
u^{p}(m, n) \leq & a(m, n)+\sum_{s=m_{0}}^{m-1} b(s, n) u^{p}(s, n)+ \\
& \sum_{s=\alpha\left(m_{0}\right)}^{\alpha(m)-1} \sum_{t=\beta(n)+1}^{\infty}\left[f(s, t) u^{q}(s, t)+g(s, t) u^{r}(s, t)+h(s, t)+\sum_{\xi=0}^{s} \sum_{\eta=t}^{\infty} w(\xi, \eta) u^{l}(\xi, \eta)\right],
\end{aligned}
$$

where $u, a, b, f, g, h, w \in \wp_{+}(\Omega)$ with $a(m, n)$ not equivalent to zero, and $f, g, h, w$ are nondecreasing in the first variable, while decreasing in the second variable, $a$ is nondecreasing in the first variable, and $b$ is decreasing in the second variable, $\alpha, \beta, p$, $q, r, l$ are defined as in Theorem 2.1. 
Theorem 2.5. If for $(m, n) \in \Omega, u(m, n)$ satisfies (12), then we have

$$
\begin{aligned}
u(m, n) \leq & \left\{\left\{a(m, n)+\widetilde{H}(m, n) \prod_{s=m_{0}}^{m-1}\left\{1+\sum_{t=n+1}^{\infty}[\alpha(s+1)-\alpha(s)][\beta(t)-\beta(t-1)]\right.\right.\right. \\
& {\left.\left.\left.\left[\tilde{f}(s, t) \frac{q}{p} K^{\frac{q-p}{p}}+\widetilde{g}(s, t) \frac{r}{p} K \frac{r-p}{p}+\sum_{\xi=0}^{s} \sum_{\eta=t}^{\infty} \widetilde{w}(\xi, \eta) \frac{l}{p} K^{\frac{l-p}{p}}\right]\right\}\right\} J(m, n)\right\}^{\frac{1}{p}} }
\end{aligned}
$$

provided $\tilde{H}(m . n)>0$, where $K>0$ is a constant, and

$$
\left\{\begin{aligned}
\tilde{H}(m, n)= & \sum_{s=\alpha\left(m_{0}\right)}^{\alpha(m)-1} \sum_{t=\beta(n)+1}^{\infty}\left\{f(s, t)\left[\frac{q}{p} K^{\frac{q-p}{p}} a(s, t) J(s, t)+\frac{p-q}{p} K^{\frac{q}{p}}\right]\right. \\
& \left.+g(s, t)\left[\frac{r}{p} K^{\frac{r-p}{p}} a(s, t)\right) J(s, t)+\frac{p-r}{p} K^{\frac{r}{p}}\right] \\
& \left.+h(s, t)+\sum_{\xi=0}^{s} \sum_{\eta=t}^{\infty} w(\xi, \eta)\left[\frac{l}{p} K^{\frac{l-p}{p}} a(\xi, \eta) J(\xi, \eta)+\frac{p-l}{p} K^{\frac{l}{p}}\right]\right\} \\
\tilde{f}(m, n)= & f(m, n) J^{\frac{q}{p}}(m, n), \tilde{g}(m, n)=g(m, n) J^{\frac{r}{p}}(m, n), \tilde{w}(m, n)=w(m, n) J^{\frac{l}{p}}(m, n), \\
J(m, n)= & \prod_{s=m_{0}}^{m-1}[1+b(s, n)] .
\end{aligned}\right.
$$

Proof: Denote $z(m, n)=\sum_{s=\alpha\left(m_{0}\right)}^{\alpha(m)-1} \sum_{t=\beta(n)+1}^{\infty}\left[f(s, t) u^{q}(s, t)+g(s, t) u^{r}(s, t)+h(s, t)+\sum_{\xi=0}^{s} \sum_{\eta=t}^{\infty} w(\xi, \eta) u^{l}(\xi, \eta)\right]$, and $v(m, n)$ $=a(m, n)+z(m, n)$. Then $v(m, n)$ is nondecreasing in the first variable, and

$$
u^{p}(m, n) \leq v(m, n)+\sum_{s=m_{0}}^{m-1} b(s, n) u^{p}(s, n)
$$

By Lemma 2.2 we obtain

$$
u^{p}(m, n) \leq v(m, n) \prod_{s=m_{0}}^{m-1}[1+b(s, n)]=(a(m, n)+z(m, n)) J(m, n)
$$

where $J(m, n)$ is defined in (14). Furthermore, using Lemma 2.1 we have

$$
\begin{aligned}
z(m, n) \leq & \sum_{s=\alpha\left(m_{0}\right)}^{\alpha(m)-1} \sum_{t=\beta(n)+1}^{\infty}\left\{f(s, t)[(a(s, t)+z(s, t)) J(s, t)]^{\frac{q}{p}}+g(s, t)[(a(s, t)+z(s, t)) J(s, t)]^{\frac{r}{p}}\right. \\
& \left.+h(s, t)+\sum_{\xi=0}^{s} \sum_{\eta=t}^{\infty} w(\xi, \eta)[(a(\xi, \eta)+z(\xi, \eta)) J(\xi, \eta)]^{\frac{l}{p}}\right\} \\
\leq & \sum_{s=\alpha\left(m_{0}\right)}^{\alpha(m)-1} \sum_{t=\beta(n)+1}^{\infty}\left\{f(s, t) J^{\frac{q}{p}}(s, t)\left[\frac{q}{p} K^{\frac{q-p}{p}}(a(s, t)+z(s, t))+\frac{p-q}{p} K^{\frac{q}{p}}\right]\right. \\
& +g(s, t) J^{\frac{r}{p}}(s, t)\left[\frac{r}{p} K^{\frac{r-p}{p}}(a(s, t)+z(s, t))+\frac{p-r}{p} K^{\frac{r}{p}}\right] \\
& \left.+h(s, t)+\sum_{\xi=0}^{s} \sum_{\eta=t}^{\infty} w(\xi, \eta) J^{\frac{l}{p}}(\xi, \eta)\left[\frac{l}{p} K^{\frac{l-p}{p}}(a(\xi, \eta)+z(\xi, \eta))+\frac{p-l}{p} K^{\frac{l}{p}}\right]\right\} \\
= & \widetilde{H}(m, n)+\sum_{s=\alpha\left(m_{0}\right)}^{\alpha(m)-1} \sum_{t=\beta(n)+1}^{\infty}\left\{\tilde{f}(s, t) \frac{q}{p} K^{\frac{q-p}{p}} z(s, t) \frac{r}{p}+\widetilde{g}(s, t) K^{\frac{r-p}{p}} z(s, t)\right. \\
& +h(s, t)+\sum_{\xi=0}^{s} \sum_{\eta=t}^{\infty} \widetilde{w}(\xi, \eta)\left[\frac{l}{p} K^{\frac{l-p}{p}} z(\xi, \eta)\right\},
\end{aligned}
$$

where $\tilde{H}, \widetilde{f}, \widetilde{g}, \widetilde{w}$ are defined in (14). 
Obviously, $\widetilde{f}, \widetilde{g}, \widetilde{w}$ are nondecreasing in the first variable, while decreasing in the second variable. Following in a same manner as the proof of Theorem 2.1 we obtain

$$
\begin{aligned}
z(m, n) \leq & \tilde{H}(m, n) \prod_{s=m_{0}}^{m-1}\left\{1+\sum_{t=n+1}^{\infty}[\alpha(s+1)-\alpha(s)][\beta(t)-\beta(t-1)]\right. \\
& {\left.\left[\tilde{f}(s, t) \frac{q}{p} K^{\frac{q-p}{p}}+\widetilde{g}(s, t) \frac{r}{p} K^{\frac{r-p}{p}}+\sum_{\xi=0}^{s} \sum_{\eta=t}^{\infty} \widetilde{w}(\xi, \eta) \frac{l}{p} K^{\frac{l-p}{p}}\right]\right\} . }
\end{aligned}
$$

Combining (16) and (18) we obtain the desired result.

Remark 4. If we take $\Omega=\mathbb{N}_{0} \times \mathbb{N}_{0}, w(m, n) \equiv 0, \alpha(m)=m, \beta(n)=n$, and omit the conditions " $f, g, h, w$ are nondecreasing in the first variable, while decreasing in the second variable" and " $b$ is decreasing in the second variable" in Theorem 2.5 , then Theorem 2.5 reduces to [[14], Theorem 7]. Furthermore, if $g(m, n) \equiv 0, q=1, p \geq 1$, then Theorem 2.5 reduces to [[13], Theorem 3].

Following a almost same process as the proof of Theorem 2.5, we have the following two theorems.

Theorem 2.6. Suppose $u, a, b, f, g, h, w \in \wp_{+}(\Omega)$ with $a(m, n)$ not equivalent to zero, and $f, g, h, w$ are decreasing both in the first variable and the second variable, $a$ is decreasing in the first variable, and $b$ is decreasing in the second variable, $\alpha, \beta$ are defined as in Theorem 2.2, and $p, q, r l$ are defined as in Theorem 2.1. If for $(m, n) \in$ $\Omega, u(m, n)$ satisfies the following inequality

$$
\begin{aligned}
u^{p}(m, n) \leq & a(m, n)+\sum_{s=m+1}^{\infty} b(s, n) u^{p}(s, n)+ \\
& \sum_{s=\alpha(m)+1}^{\infty} \sum_{t=\beta(n)+1}^{\infty}\left[f(s, t) u^{q}(s, t)+g(s, t) u^{r}(s, t)+h(s, t)+\sum_{\xi=s}^{\infty} \sum_{\eta=t}^{\infty} w(\xi, \eta) u^{l}(\xi, \eta)\right]
\end{aligned}
$$

then we have

$$
\begin{aligned}
u(m, n) \leq & \left\{\left\{a(m, n)+\widetilde{H}(m, n) \prod_{s=m+1}^{\infty}\left\{1+\sum_{t=n+1}^{\infty}[\alpha(s+1)-\alpha(s)][\beta(t)-\beta(t-1)]\right.\right.\right. \\
& {\left.\left.\left.\left[\tilde{f}(s, t) \frac{q}{p} K^{\frac{q-p}{p}}+\widetilde{g}(s, t) \frac{r}{p} K^{\frac{r-p}{p}}+\sum_{\xi=s}^{\infty} \sum_{\eta=t}^{\infty} \widetilde{w}(\xi, \eta) \frac{l}{p} K^{\frac{l-p}{p}}\right]\right\}\right\} J(m, n)\right\}^{\frac{1}{p}} }
\end{aligned}
$$

provided $\widetilde{H}(m . n)>0$, where

$$
\left\{\begin{aligned}
\widetilde{H}(m, n)= & \sum_{s=\alpha(m)+1}^{\infty} \sum_{t=\beta(n)+1}^{\infty}\left\{f(s, t)\left[\frac{q}{p} K^{\frac{q-p}{p}} a(s, t) J(s, t)+\frac{p-q}{p} K^{\frac{q}{p}}\right]\right. \\
& \left.+g(s, t)\left[\frac{r}{p} K^{\frac{r-p}{p}} a(s, t)\right) J(s, t)+\frac{p-r}{p} K^{\frac{r}{p}}\right] \\
& \left.+h(s, t)+\sum_{\xi=s}^{\infty} \sum_{\eta=t}^{\infty} w(\xi, \eta)\left[\frac{l}{p} K^{\frac{l-p}{p}} a(\xi, \eta) J(\xi, \eta)+\frac{p-l}{p} K^{\frac{l}{p}}\right]\right\} \\
\tilde{f}(m, n)= & f(m, n) J^{\frac{q}{p}}(m, n), \widetilde{g}(m, n)=g(m, n) J^{\frac{r}{p}}(m, n), \widetilde{w}(m, n)=w(m, n) J^{\frac{l}{p}}(m, n), \\
J(m, n)= & \prod_{s=m+1}^{\infty}[1+b(s, n)] .
\end{aligned}\right.
$$

Theorem 2.7. Suppose $u, a, b, f, g, h, w \in \wp_{+}(\Omega)$ with $a(m, n)$ not equivalent to zero, and $f, g, h, w$ are decreasing both in the first variable and the second variable, $a$ 
is nondecreasing in the first variable, and $b$ is decreasing in the second variable, $\alpha, \beta$ are defined as in Theorem 2.2, and $p, q, r, l$ are defined as in Theorem 2.1. If for $(m$, $n) \in \Omega, u(m, n)$ satisfies the following inequality

$$
\begin{aligned}
u^{p}(m, n) \leq & a(m, n)+\sum_{s=m_{0}}^{m-1} b(s, n) u^{p}(s, n)+ \\
& \sum_{s=\alpha(m)+1}^{\infty} \sum_{t=\beta(n)+1}^{\infty}\left[f(s, t) u^{q}(s, t)+g(s, t) u^{r}(s, t)+h(s, t)+\sum_{\xi=s}^{\infty} \sum_{\eta=t}^{\infty} w(\xi, \eta) u^{l}(\xi, \eta)\right]
\end{aligned}
$$

then we have

$$
\begin{gathered}
u(m, n) \leq\left\{\left\{a(m, n)+\widetilde{H}(m, n) \prod_{s=m+1}^{\infty}\left\{1+\sum_{t=n+1}^{\infty}[\alpha(s+1)-\alpha(s)][\beta(t)-\beta(t-1)]\right.\right.\right. \\
\left.\left.\left.\left[\widetilde{f}(s, t) \frac{q}{p} K^{\frac{q-p}{p}}+\widetilde{g}(s, t) \frac{r}{p} K^{\frac{r-p}{p}}+\sum_{\xi=s}^{\infty} \sum_{\eta=t}^{\infty} \widetilde{w}(\xi, \eta) \frac{l}{p} K^{\frac{l-p}{p}}\right]\right\}\right\} J(m, n)\right\}^{\frac{1}{p}}
\end{gathered}
$$

provided $\widetilde{H}(m . n)>0$, where $K>0$ is a constant, and

$$
\begin{aligned}
\widetilde{H}(m, n) & =\sum_{s=\alpha(m)+1}^{\infty} \sum_{t=\beta(n)+1}^{\infty}\left\{f(s, t)\left[\frac{q}{p} K^{\frac{q-p}{p}} a(s, t) J(s, t)+\frac{p-q}{p} K^{\frac{q}{p}}\right]\right. \\
& \left.+g(s, t)\left[\frac{r}{p} K^{\frac{r-p}{p}} a(s, t)\right) J(s, t)+\frac{p-r}{p} K^{\frac{r}{p}}\right] \\
& \left.+h(s, t)+\sum_{\xi=s}^{\infty} \sum_{\eta=t}^{\infty} w(\xi, \eta)\left[\frac{l}{p} K^{\frac{l-p}{p}} a(\xi, \eta) J(\xi, \eta)+\frac{p-l}{p} K^{\frac{l}{p}}\right]\right\},
\end{aligned}
$$

and $\tilde{f}(m, n), \widetilde{g}(m, n), \widetilde{w}(m, n), J(m, n)$ are defined as in Theorem 2.5 .

Remark 5. If we take $\Omega=\mathbb{N}_{0} \times \mathbb{N}_{0}, w(m, n) \equiv 0, \alpha(m)=m, \beta(n)=n$, and omit the conditions " $f, g, h, w$ are decreasing both in the first variable and the second variable" and " $b$ is decreasing in the second variable" in Theorem 2.6, then Theorem 2.6 reduces to [[14], Theorem 8]. Furthermore, if $g(m, n) \equiv 0, q=1, p \geq 1$, then Theorem 2.6 reduces to [[13], Theorem 4].

Remark 6. If we take $\Omega=\mathbb{N}_{0} \times \mathbb{N}_{0}, w(m, n) \equiv 0, \alpha(m)=m, \beta(n)=n$, and omit the conditions " $f, g, h, w$ are decreasing both in the first variable and the second variable" and " $b$ is decreasing in the second variable" in Theorem 2.7, then Theorem 2.7 reduces to [[12], Theorem $2.7(\mathrm{q} 1)]$.

In the following, we will study the difference inequality with the following form

$$
u^{p}(m, n) \leq a(m, n)+\sum_{s=m_{0}}^{m-1} b(s, n) u^{p}(s, n)+\sum_{s=\alpha\left(m_{0}\right)}^{\alpha(m)-1} \sum_{t=\beta(n)+1}^{\infty}\left[L(s, t, u(s, t))+\sum_{\xi=0}^{s} \sum_{\eta=t}^{\infty} w(\xi, \eta) u^{l}(\xi, \eta)\right]
$$

where $u, a, b, w \in \wp_{+}(\Omega)$ with $a(m, n)$ not equivalent to zero, and $w$ is nondecreasing in the first variable, while decreasing in the second variable, $a$ is nondecreasing in the first variable, and $b$ is decreasing in the second variable, $\alpha, \beta$ are defined as in Theorem 2.1, $L: \Omega \times \mathbb{R}_{+} \rightarrow \mathbb{R}_{+}$satisfies $0 \leq L(m, n, u)-L(m, n, v) \leq$ $M(m, n, v)(u-v)$ for $u \geq v \geq 0$, where $M: \Omega \times \mathbb{R}_{+} \rightarrow \mathbb{R}_{+} \cdot p, l$ are defined as in Theorem 2.1 with $p \geq 1$. 
Theorem 2.8. If for $(m, n) \in \Omega, u(m, n)$ satisfies (19), then

$$
\begin{aligned}
u(m, n) \leq & \left\{\left\{a(m, n)+\widehat{H}(m, n) \prod_{s=m_{0}}^{m-1}\left\{1+\sum_{t=n+1}^{\infty}[\alpha(s+1)-\alpha(s)][\beta(t)-\beta(t-1)]\right.\right.\right. \\
& \left.\left.\left.\left.\widehat{f}(s, t) \frac{1}{p} K^{\frac{1-p}{p}}+\sum_{\xi=0}^{s} \sum_{\eta=t}^{\infty} \widehat{w}(\xi, \eta) \frac{l}{p} K^{\frac{l-p}{p}}\right]\right\}\right\} J(m, n)\right\}^{\frac{1}{p}},
\end{aligned}
$$

provided that $\widehat{H}(m . n)>0$, and $\widehat{f}(m, n)$ is nondecreasing in the first variable and decreasing in the second variable, where $K>0$ is a constant, and

$$
\left\{\begin{aligned}
\widehat{H}(m, n)= & \sum_{s=\alpha\left(m_{0}\right)}^{\alpha(m)-1} \sum_{t=\beta(n)+1}^{\infty}\left\{L\left(s, t, J^{\frac{1}{p}}(s, t)\left(\frac{1}{p} K^{\frac{1-p}{p}} a(s, t)+\frac{p-1}{p} K^{\frac{1}{p}}\right)\right)\right. \\
& \left.+\sum_{\xi=0}^{s} \sum_{\eta=t}^{\infty} w(\xi, \eta) J^{\frac{l}{p}}(\xi, \eta)\left[\frac{l}{p} K^{\frac{l-p}{p}} a(\xi, \eta)+\frac{p-l}{p} K^{\frac{l}{p}}\right]\right\} \\
\widehat{f}(m, n)= & M\left(m, n, J^{\frac{1}{p}}(m, n)\left(\frac{1}{p} K^{\frac{1-p}{p}} a(m, n)+\frac{p-1}{p} K^{\frac{1}{p}}\right)\right) J^{\frac{1}{p}}(m, n) \frac{1}{p} K^{\frac{1-p}{p}} \\
\widehat{w}(m, n)= & w(m, n) J^{\frac{l}{p}}(m, n), J(m, n)=\prod_{s=m_{0}}^{m-1}[1+b(s, n)] .
\end{aligned}\right.
$$

Proof: Denote $z(m, n)=\sum_{s=\alpha\left(m_{0}\right)}^{\alpha(m)-1} \sum_{t=\beta(n)+1}^{\infty}\left[L(s, t, u(s, t))+\sum_{\xi=0}^{s} \sum_{\eta=t}^{\infty} w(\xi, \eta) u^{l}(\xi, \eta)\right]$, and $v(m, n)$ $=a(m, n)+z(m, n)$. Then $v(m, n)$ is nondecreasing in the first variable, and

$$
u^{p}(m, n) \leq v(m, n)+\sum_{s=m_{0}}^{m-1} b(s, n) u^{p}(s, n)
$$

By Lemma 2.2 we obtain

$$
u^{p}(m, n) \leq v(m, n) \prod_{s=m_{0}}^{m-1}[1+b(s, n)]=(a(m, n)+z(m, n)) J(m, n),
$$

where $J(m, n)$ is defined in (21). Furthermore,

$$
\begin{aligned}
z(m, n) \leq & \sum_{s=\alpha\left(m_{0}\right)}^{\alpha(m)-1} \sum_{t=\beta(n)+1}^{\infty}\left\{L\left(s, t,((a(s, t)+z(s, t)) J(s, t))^{\frac{1}{p}}\right)+\sum_{\xi=0}^{s} \sum_{\eta=t}^{\infty} w(\xi, \eta)((a(\xi, \eta)+z(\xi, \eta)) J(\xi, \eta))^{\frac{1}{p}}\right\} \\
\leq & \sum_{s=\alpha\left(m_{0}\right)}^{\alpha(m)-1} \sum_{t=\beta(n)+1}^{\infty}\left\{L\left(s, t, J^{\frac{1}{p}}(s, t)\left(\frac{1}{p} K^{\frac{1-p}{p}}(a(s, t)+z(s, t))+\frac{p-1}{p} K^{\frac{1}{p}}\right)\right)\right. \\
& \left.+\sum_{\xi=0}^{s} \sum_{\eta=t}^{\infty} w(\xi, \eta) J^{\frac{l}{p}}(\xi, \eta)\left(\frac{l}{p} K^{\frac{l-p}{p}}(a(\xi, \eta)+z(\xi, \eta))+\frac{p-l}{p} K^{\frac{1}{p}}\right)\right\} \\
= & \sum_{s=\alpha\left(m_{0}\right)}^{\alpha(m)-1} \sum_{t=\beta(n)+1}^{\infty}\left\{L\left(s, t, J^{\frac{1}{p}}(s, t)\left(\frac{1}{p} K^{\frac{1-p}{p}}(a(s, t)+z(s, t))+\frac{p-1}{p} K^{\frac{1}{p}}\right)\right)\right. \\
& -L\left(s, t, J^{\frac{1}{p}}(s, t)\left(\frac{1}{p} K^{\frac{1-p}{p}} a(s, t)+\frac{p-1}{p} K^{\frac{1}{p}}\right)\right)+L\left(s, t, J^{\frac{1}{p}}(s, t)\left(\frac{1}{p} K^{\frac{1-p}{p}} a(s, t)+\frac{p-1}{p} K^{\frac{1}{p}}\right)\right) \\
& \left.+\sum_{\xi=0}^{s} \sum_{\eta=t}^{\infty} w(\xi, \eta) J^{\frac{1}{p}}(\xi, \eta)\left[\frac{l}{p} K^{\frac{1-p}{p}}(a(\xi, \eta)+z(\xi, \eta))+\frac{p-l}{p} K^{\frac{l}{p}}\right]\right\} \\
\leq & \sum_{s=\alpha\left(m_{0}\right)} \sum_{t=\beta(n)+1}^{\infty}\left\{M\left(s, t, J^{\frac{1}{p}}(s, t)\left(\frac{1}{p} K^{\frac{1-p}{p}} a(s, t)+\frac{p-1}{p} K^{\frac{1}{p}}\right)\right) J^{\frac{1}{p}}(s, t) \frac{1}{p} K^{\frac{1-p}{p}} z(s, t)\right. \\
& +L\left(s, t, J^{\frac{1}{p}}(s, t)\left(\frac{1}{p} K^{\frac{1-p}{p}} a(s, t)+\frac{p-1}{p} K^{\frac{1}{p}}\right)\right) \\
& \left.+\sum_{\xi=0}^{s} \sum_{\eta=t}^{\infty} w(\xi, \eta) J^{\frac{1}{p}}(\xi, \eta)\left[\frac{l}{p} K^{\frac{l-p}{p}}(a(\xi, \eta)+z(\xi, \eta))+\frac{p-l}{p} K^{\frac{1}{p}}\right]\right\} \\
= & \widehat{H}(m, n)+\sum_{s=\alpha\left(m_{0}\right)}^{\alpha(m)-1} \sum_{t=\beta(n)+1}^{\infty}\left\{\widehat{f}(s, t) z(s, t)+\sum_{\xi=0}^{s} \sum_{\eta=t}^{\infty} \widehat{w}(\xi, \eta) \frac{l}{p} K^{\frac{l-p}{p}} z(\xi, \eta)\right\},
\end{aligned}
$$


where $\widehat{H}, \widehat{f}, \widehat{w}$ are defined in (21).

Then following in a same manner as the proof of Theorem 2.1 we obtain

$$
z(m, n) \leq \widehat{H}(m, n) \prod_{s=m_{0}}^{m-1}\left\{1+\sum_{t=n+1}^{\infty}[\alpha(s+1)-\alpha(s)][\beta(t)-\beta(t-1)]\left[\widehat{f}(s, t) \frac{1}{p} K^{\frac{1-p}{p}}+\sum_{\xi=0}^{s} \sum_{\eta=t}^{\infty} \widehat{w}(\xi, \eta) \frac{l}{p} K^{\frac{l-p}{p}}\right]\right\} .
$$

The desired inequality can be deduced by the combination of (23) and (25).

Theorem 2.9. Suppose $u, a, b, w \in \wp_{+}(\Omega)$ with $a(m, n)$ not equivalent to zero, and $w$ is decreasing both in the first variable and the second variable, $a$ is decreasing in the first variable, and $b$ is decreasing in the second variable, $\alpha, \beta$ are defined as in Theorem 2.2, and $L$ is defined as in Theorem 2.8. $p, l$ are defined as in Theorem 2.1 with $p \geq 1$. If for $(m, n) \in \Omega, u(m, n)$ satisfies the following inequality

$$
u^{p}(m, n) \leq a(m, n)+\sum_{s=m+1}^{\infty} b(s, n) u^{p}(s, n)+\sum_{s=\alpha(m)+1}^{\infty} \sum_{t=\beta(n)+1}^{\infty}\left[L(s, t, u(s, t))+\sum_{\xi=s}^{\infty} \sum_{\eta=t}^{\infty} w(\xi, \eta) u^{l}(\xi, \eta)\right]
$$

then

$$
\begin{aligned}
u(m, n) \leq & \left\{\left\{a(m, n)+\widehat{H}(m, n) \prod_{s=m+1}^{\infty}\left\{1+\sum_{t=n+1}^{\infty}[\alpha(s+1)-\alpha(s)][\beta(t)-\beta(t-1)]\right.\right.\right. \\
& \left.\left.\left.\left.\widehat{f}(s, t) \frac{1}{p} K^{\frac{1-p}{p}}+\sum_{\xi=s}^{\infty} \sum_{\eta=t}^{\infty} \widehat{w}(\xi, \eta) \frac{l}{p} K^{\frac{l-p}{p}}\right]\right\}\right\} J(m, n)\right\}^{\frac{1}{p}}
\end{aligned}
$$

provided that $\widehat{H}(m . n)>0$, and $\widehat{f}(m, n)$ is decreasing both in the first variable and the second variable, where

$$
\left\{\begin{aligned}
\widehat{H}(m, n)= & \sum_{s=\alpha(m)+1}^{\infty} \sum_{t=\beta(n)+1}^{\infty}\left\{L\left(s, t, J^{\frac{1}{p}}(s, t)\left[\frac{1}{p} K^{\frac{1-p}{p}} a(s, t)+\frac{p-1}{p} K^{\frac{1}{p}}\right]\right)\right. \\
& \left.+\sum_{\xi=s}^{\infty} \sum_{\eta=t}^{\infty} w(\xi, \eta) J^{\frac{l}{p}}(\xi, \eta)\left[\frac{l}{p} K^{\frac{l-p}{p}} a(\xi, \eta)+\frac{p-l}{p} K^{\frac{1}{p}}\right]\right\}, \\
\widehat{f}(m, n)= & M\left(m, n, J^{\frac{1}{p}}(m, n)\left(\frac{1}{p} K^{\frac{1-p}{p}} a(m, n)+\frac{p-1}{p} K^{\frac{1}{p}}\right)\right) J^{\frac{1}{p}}(m, n) \frac{1}{p} K^{\frac{1-p}{p}} \\
\widehat{w}(m, n)= & w(m, n) J^{\frac{l}{p}}(m, n), J(m, n)=\prod_{s=m+1}^{\infty}[1+b(s, n)] .
\end{aligned}\right.
$$

The proof for Theorem 2.8 is similar to Theorem 2.7, and we omit it here.

Remark 7. If we take $\Omega=\mathbb{N}_{0} \times \mathbb{N}_{0}, w(m, n) \equiv 0, \alpha(m)=m, \beta(n)=n$, and omit the conditions " $w$ is nondecreasing in the first variable, while decreasing in the second variable", " $\widehat{f}(m, n)$ is nondecreasing in the first variable and decreasing in the second variable", and " $b$ is decreasing in the second variable" in Theorem 2.8 , then Theorem 2.8 reduces to [[13], Theorem 5].

Remark 8. If we take $\Omega=\mathbb{N}_{0} \times \mathbb{N}_{0}, w(m, n) \equiv 0, \alpha(m)=m, \beta(n)=n$, and omit the conditions " $w$ is decreasing both in the first variable and the second variable", " $\widehat{f}(m, n)$ is decreasing both in the first variable and the second variable" and " $b$ is decreasing in the second variable" in Theorem 2.9, then Theorem 2.9 reduces to [[13], Theorem 6].

\section{Applications}

In this section, we will present some applications for the established results above, and show they are useful in the study of boundedness, uniqueness, continuous dependence of solutions of certain difference equations. 
Example 1. Consider the following difference equation

$$
-\Delta_{12} u^{p}(m, n)=F_{1}(m, n+1, u(m, n+1))+\sum_{\xi=m_{0}}^{m} \sum_{\eta=n+1}^{\infty} F_{2}(\xi, \eta, u(\xi, \eta)),
$$

with the initial condition

$$
u^{p}(m, \infty)=f(m), u^{p}\left(m_{0}, n\right)=g(n), f\left(m_{0}\right)=g(\infty)=C,
$$

where $p \geq 1$ is an odd number, $u \in \wp(\Omega), F_{1}, F_{2}: \Omega \times \mathbb{R} \rightarrow \mathbb{R}$.

Theorem 3.1. Suppose $u(m, n)$ is a solution of (26) and (27). If $|f(m)+g(n)-C| \leq \sigma$, $\left|F_{1}(m, n, u)\right| \leq f_{1}(m, n)|u|$, and $\left|F_{2}(m, n, u)\right| \leq f_{2}(m, n)|u|$, where $f_{1}, f_{2} \in \wp_{+}(\Omega)$, then we have

$$
|u(m, n)| \leq\left\{\sigma+H(m, n) \prod_{s=m_{0}}^{m-1}\left\{1+\sum_{t=n+1}^{\infty}\left[f_{1}(s, t) \frac{1}{p} K^{\frac{1-p}{p}}+\sum_{\xi=0}^{s} \sum_{\eta=t}^{\infty} f_{2}(\xi, \eta) \frac{1}{p} K^{\frac{1-p}{p}}\right]\right\}\right\}^{\frac{1}{p}},
$$

where $K>0$ is a constant, and

$$
H(m, n)=\sum_{s=m_{0}}^{m-1} \sum_{t=n+1}^{\infty}\left\{f_{1}(s, t)\left[\frac{1}{p} K^{\frac{1-p}{p}} \sigma+\frac{p-1}{p} K^{\frac{1}{p}}\right]+\sum_{\xi=0}^{s} \sum_{\eta=t}^{\infty} f_{2}(\xi, \eta)\left[\frac{1}{p} K^{\frac{1-p}{p}} \sigma+\frac{p-1}{p} K^{\frac{1}{p}}\right]\right\} .
$$

Proof. The equivalent form of (26) and (27) is denoted by

$$
u^{p}(m, n)=f(m)+g(n)-C+\sum_{s=m_{0}}^{m-1} \sum_{t=n+1}^{\infty}\left[F_{1}(s, t, u(s, t))+\sum_{\xi=0}^{s} \sum_{\eta=t}^{\infty} F_{2}(\xi, \eta, u(\xi, \eta))\right] .
$$

Then we have

$$
\begin{aligned}
|u(m, n)|^{p} & \leq|f(m)+g(n)-C|+\sum_{s=m_{0}}^{m-1} \sum_{t=n+1}^{\infty}\left|F_{1}(s, t, u(s, t))+\sum_{\xi=0}^{s} \sum_{\eta=t}^{\infty} F_{2}(\xi, \eta, u(\xi, \eta))\right| \\
& \leq|f(m)+g(n)-C|+\sum_{s=m_{0}}^{m-1} \sum_{t=n+1}^{\infty}\left|F_{1}(s, t, u(s, t))\right|+\sum_{\xi=0}^{s} \sum_{\eta=t}^{\infty}\left|F_{2}(\xi, \eta, u(\xi, \eta))\right| \\
& \leq \sigma+\sum_{s=m_{0}}^{m-1} \sum_{t=n+1}^{\infty} f_{1}(s, t)|u(s, t)|+\sum_{\xi=0}^{s} \sum_{\eta=t}^{\infty} f_{2}(\xi, \eta)|u(\xi, \eta)| .
\end{aligned}
$$

We note that it is unnecessary for $f_{1}, f_{2}$ being nondecreasing or decreasing since $\alpha(m)=$ $m, \beta(n)=n$ here, and a suitable application of Theorem 2.1 to (31) yields the desired result.

The following theorem deals with the uniqueness of solutions of (26) and (27).

Theorem 3.2. Suppose $\left|F_{i}(m, n, u)-F_{i}(m, n, v)\right| \leq f_{i}(m, n)\left|u^{p}-v^{p}\right|, i=1,2$, where $f_{i}$ $\in \wp_{+}(\Omega), i=1,2$, then (26) and (27) has at most one solution.

Proof. Suppose $u_{1}(m, n), u_{2}(m, n)$ are two solutions of (26) and (27). Then

$$
\begin{aligned}
& \left|u_{1}^{p}(m, n)-u_{2}^{p}(m, n)\right| \\
= & \left|\sum_{s=m_{0}}^{m-1} \sum_{t=n+1}^{\infty} F_{1}\left(s, t, u_{1}(s, t)\right)-F_{1}\left(s, t, u_{2}(s, t)\right)+\sum_{\xi=0}^{s} \sum_{\eta=t}^{\infty}\left[F_{2}\left(\xi, \eta, u_{1}(\xi, \eta)\right)-F_{2}\left(\xi, \eta, u_{2}(\xi, \eta)\right)\right]\right| \\
\leq & \sum_{s=m_{0}}^{m-1} \sum_{t=n+1}^{\infty}\left|F_{1}\left(s, t, u_{1}(s, t)\right)-F_{1}\left(s, t, u_{2}(s, t)\right)\right|+\sum_{\xi=0}^{s} \sum_{\eta=t}^{\infty}\left|F_{2}\left(\xi, \eta, u_{1}(\xi, \eta)\right)-F_{2}\left(\xi, \eta, u_{2}(\xi, \eta)\right)\right| \\
\leq & \sum_{s=m_{0}}^{m-1} \sum_{t=n+1}^{\infty}\left|f_{1}(s, t)\right| u_{1}^{p}(s, t)-u_{2}^{p}(s, t)\left|+\sum_{\xi=0}^{s} \sum_{\eta=t}^{\infty}\right| f_{2}(\xi, \eta)\left|u_{1}^{p}(\xi, \eta)-u_{2}^{p}(\xi, \eta)\right|
\end{aligned}
$$

Treat $\left|u_{1}^{p}(m, n)-u_{2}^{p}(m, n)\right|$ as one variable, and a suitable application of Theorem 2.1 to (32) yields $\left|u_{1}^{p}(m, n)-u_{2}^{p}(m, n)\right| \leq 0$, which implies $u_{1}^{p}(m, n) \equiv u_{2}^{p}(m, n)$. 
Since $p$ is an odd number, then we have $u_{1}(m, n) \equiv u_{2}(m, n)$, and the proof is complete.

The following theorem deals with the continuous dependence of the solution of (26) and (27) on the functions $F_{1}, F_{2}$ and the initial value $f(m), g(n)$.

Theorem 3.3. Assume $\left|F_{i}\left(m, n, u_{1}\right)-F_{i}\left(s, t, u_{2}\right)\right| \leq f_{i}(s, t)\left|u_{1}^{p}-u_{2}^{p}\right|, i=1,2$, where $f_{i} \in \wp_{+}(\Omega), i=1,2, \mid f(m)-\bar{f}(m)+g(n)-\bar{g}(n) \leq \varepsilon$, where $\varepsilon>0$ is a constant, and furthermore, assume $\quad \sum_{s=n_{0}}^{m-1} \sum_{l=n+1}^{\infty}\left\{\left[F_{1}(s, t, \bar{u}(s, t))-\bar{F}_{1}(s, t, \bar{u}(s, t))+\sum_{\xi=0}^{s} \sum_{\eta=t}^{\infty}\left|F_{2}(\xi, \eta, \bar{u}(\xi, \eta))-\bar{F}_{2}(\xi, \eta, \bar{u}(\xi, \eta))\right|\right\} \leq \varepsilon\right.$, $\bar{u} \in \wp(\Omega)$ is the solution of the following difference equation

$$
-\Delta_{12} \bar{u}^{p}(m, n)=\bar{F}_{1}(m, n+1, \bar{u}(m, n+1))+\sum_{\xi=0}^{m} \sum_{\eta=n+1}^{\infty} \bar{F}_{2}(\xi, \eta, \bar{u}(\xi, \eta)),
$$

with the initial condition

$$
\bar{u}^{p}(m, \infty)=\bar{f}(m), \bar{u}^{p}\left(m_{0}, n\right)=\bar{g}(n), \bar{f}\left(m_{0}\right)=\bar{g}(\infty)=C,
$$

where $\bar{F}_{1}, \bar{F}_{2}: \Omega \times \mathbb{R} \rightarrow \mathbb{R}$, then

$$
\left|u^{p}(m, n)-\bar{u}^{p}(m, n)\right| \leq(2 \varepsilon)^{\frac{1}{p}} K,
$$

provided that $G(m, n) \leq K$, where

$$
G(m, n)=\left\{1+\left\{\sum_{s=m_{0}}^{m-1} \sum_{t=n=1}^{\infty}\left[f_{1}(s, t)+\sum_{\xi=0}^{s} \sum_{\eta=t}^{\infty} f_{2}(\xi, \eta)\right]\right\} \prod_{s=m_{0}}^{m-1}\left\{1+\sum_{t=n=1}^{\infty}\left[f_{1}(s, t)+\sum_{\xi=0}^{s} \sum_{\eta=t}^{\infty} f_{2}(\xi, \eta)\right]\right\}\right\}^{\frac{1}{p}} .
$$

Proof. The equivalent form of (33) and (34) is denoted by

$$
\bar{u}^{p}(m, n)=\bar{f}(m)+\bar{g}(n)-C+\sum_{s=m_{0}}^{m-1} \sum_{t=n+1}^{\infty}\left[\bar{F}_{1}(s, t, \bar{u}(s, t))+\sum_{\xi=0}^{s} \sum_{\eta=t}^{\infty} \bar{F}_{2}(\xi, \eta, \bar{u}(\xi, \eta))\right] .
$$

Then from (30) and (36) we have

$$
\begin{aligned}
& \left|u^{p}(m, n)-\bar{u}^{p}(m, n)\right| \\
= & \mid f(m)+g(n)-C+\sum_{s=m_{0}}^{m-1} \sum_{t=n+1}^{\infty}\left[F_{1}(s, t, u(s, t))+\sum_{\xi=0}^{s} \sum_{\eta=t}^{\infty} F_{2}(\xi, \eta, u(\xi, \eta))\right] \\
& -\bar{f}(m)-\bar{g}(n)+C-\sum_{s=m_{0}}^{m-1} \sum_{t=n+1}^{\infty}\left[\bar{f}_{1}(s, t, \bar{u}(s, t))-\sum_{\xi=0}^{s} \sum_{\eta=t}^{\infty} \bar{f}_{2}(\xi, \eta, \bar{u}(\xi, \eta))\right] \mid \\
\leq & |f(m)-\bar{f}(m)+g(n)-\bar{g}(n)|+\sum_{s=m_{0}}^{m-1} \sum_{t=n+1}^{\infty}\left\{\left|F_{1}(s, t, u(s, t))-\bar{f}_{1}(s, t, \bar{u}(s, t))\right|\right. \\
& \left.+\sum_{\xi=0}^{s} \sum_{\eta=t}^{\infty}\left|F_{2}(\xi, \eta, u(\xi, \eta))-\bar{f}_{2}(\xi, \eta, \bar{u}(\xi, \eta))\right|\right\} \\
\leq & \varepsilon+\sum_{s=m_{0}}^{m-1} \sum_{t=n+1}^{\infty}\left\{\left|F_{1}(s, t, u(s, t))-F_{1}(s, t, \bar{u}(s, t))\right|+\left|F_{1}(s, t, \bar{u}(s, t))-\bar{f}_{1}(s, t, \bar{u}(s, t))\right|\right. \\
& \left.+\sum_{\xi=0}^{s} \sum_{\eta=t}^{\infty}\left|F_{2}(\xi, \eta, u(\xi, \eta))-F_{2}(\xi, \eta, \bar{u}(\xi, \eta))\right|+\left|F_{2}(\xi, \eta, \bar{u}(\xi, \eta))-\bar{f}_{2}(\xi, \eta, \bar{u}(\xi, \eta))\right|\right\} \\
\leq & 2 \varepsilon+\sum_{s=m_{0}}^{m-1} \sum_{t=n+1}^{\infty}\left\{\left|F_{1}(s, t, u(s, t))-F_{1}(s, t, \bar{u}(s, t))\right|+\sum_{\xi=0}^{s} \sum_{\eta=t}^{\infty}\left|F_{2}(\xi, \eta, u(\xi, \eta))-F_{2}(\xi, \eta, \bar{u}(\xi, \eta))\right|\right\} \\
\leq & 2 \varepsilon+\sum_{s=m_{0}}^{m-1} \sum_{t=n+1}^{\infty}\left\{f_{1}(s, t)\left|u^{p}(s, t)-\bar{u}^{p}(s, t)\right|+\sum_{\xi=0}^{s} \sum_{\eta=t}^{\infty} f_{2}(\xi, \eta)\left|u^{p}(\xi, \eta)-\bar{u}^{p}(\xi, \eta)\right|\right\} .
\end{aligned}
$$


Then a suitable application of Theorem 2.1 to (37) yields the desired result.

Example 2. Consider the following difference equation

$$
u^{p}(m, n)=a(m, n)+\sum_{s=m+1}^{\infty} b(s, n) u^{p}(s, n)+\sum_{s=m+1}^{\infty} \sum_{t=n+1}^{\infty}\left[F_{1}(s, t, u(s, t))+\sum_{\xi=s}^{\infty} \sum_{\eta=t}^{\infty} F_{2}(\xi, \eta, u(\xi, \eta))\right]
$$

where $u, a, b \in \wp(\Omega)$ with $a(m, n)$ not equivalent to zero, $p \geq 1$ is an odd number, $F_{1}, F_{2}: \Omega \times \mathbb{R} \rightarrow \mathbb{R}$.

Theorem 3.4. Suppose $u(m, n)$ is a solution of (38). If $\left|F_{1}(m, n, u)\right| \leq L(m, n, u), \mid F_{2}$ $\left.(m, n, u)|\leq w(m, n)| u\right|^{l}$, where $L$ is defined as in Theorem 2.8, and $w \in \wp_{+}(\Omega), l \geq 0, p$ $\geq l$, then we have

$$
|u(m, n)| \leq\left\{\left\{|a(m, n)|+\widehat{H}(m, n) \prod_{s=m+1}^{\infty}\left\{1+\sum_{t=n+1}^{\infty} \widehat{f}(s, t) \frac{1}{p} K^{\frac{1-p}{p}}+\sum_{\xi=s}^{\infty} \sum_{\eta=t}^{\infty} \widehat{w}(\xi, \eta) \frac{l}{p} K^{\frac{l-p}{p}}\right]\right\} J(m, n)\right\}^{\frac{1}{p}},
$$

where

$$
\left\{\begin{aligned}
\widehat{H}(m, n)= & \sum_{s=m+1}^{\infty} \sum_{t=n+1}^{\infty}\left\{L\left(s, t, J^{\frac{1}{p}}(s, t)\left[\frac{1}{p} K^{\frac{1-p}{p}} a(s, t)+\frac{p-1}{p} K^{\frac{1}{p}}\right]\right)\right. \\
& \left.+\sum_{\xi=s}^{\infty} \sum_{\eta=t}^{\infty} w(\xi, \eta) J^{\frac{l}{p}}(\xi, \eta)\left[\frac{l}{p} K^{\frac{l-p}{p}} a(\xi, \eta)+\frac{p-l}{p} K^{\frac{l}{p}}\right]\right\}, \\
\widehat{f}(m, n)= & M\left(m, n, J^{\frac{1}{p}}(m, n)\left(\frac{1}{p} K^{\frac{1-p}{p}} a(m, n)+\frac{p-1}{p} K^{\frac{1}{p}}\right)\right) J^{\frac{1}{p}}(m, n) \frac{1}{p} K^{\frac{1-p}{p}}, \\
\widehat{w}(m, n)= & w(m, n) J^{\frac{l}{p}}(m, n), J(m, n)=\prod_{s=m+1}^{\infty}[1+|b(s, n)|] .
\end{aligned}\right.
$$

Proof. From (38) we have

$$
\begin{aligned}
|u(m, n)|^{p} & \leq|a(m, n)|+\sum_{s=m+1}^{\infty}|b(s, n)||u(s, n)|^{p}+\sum_{s=m+1}^{\infty} \sum_{t=n+1}^{\infty}\left[\left|F_{1}(s, t, u(s, t))\right|+\sum_{\xi=s}^{\infty} \sum_{\eta=t}^{\infty}\left|F_{2}(\xi, \eta, u(\xi, \eta))\right|\right] \\
& \leq|a(m, n)|+\sum_{s=m+1}^{\infty}|b(s, n)||u(s, n)|^{p}+\sum_{s=m+1}^{\infty} \sum_{t=n+1}^{\infty}\left[L(s, t, u(s, t))+\sum_{\xi=s}^{\infty} \sum_{\eta=t}^{\infty} w(\xi, \eta)|u(\xi, \eta)|^{l}\right] .
\end{aligned}
$$

Then a suitable application of Theorem 2.9 (with $\alpha(m)=m, \beta(n)=n$ ) to (41) yields the desired result.

Similar to Theorems 3.2 and 3.3, we also have the following two theorems dealing with the uniqueness and continuous dependence of the solution of (38) on the functions $a, b, F_{1}, F_{2}$.

Theorem 3.5. Suppose $\left|F_{i}(m, n, u)-F_{i}(m, n, v)\right| \leq f_{i}(m, n)\left|u^{p}-v^{p}\right|, i=1,2$, where $f_{i}$ $\in \wp_{+}(\Omega), i=1,2$, then (38) has at most one solution.

Theorem 3.6. Assume $\left|F_{i}\left(m, n, u_{1}\right)-F_{i}\left(s, t, u_{2}\right)\right| \leq f_{i}(s, t)\left|u_{1}^{p}-u_{2}^{p}\right|, i=1,2$, where $f_{i} \in \wp_{+}(\Omega), \quad i=1,2, \mid f(m)-\bar{f}(m)+g(n)-\bar{g}(n) \leq \varepsilon$, and furthermore, assume $\bar{u} \in \wp(\Omega), \bar{u} \in \wp(\Omega)$ is the solution of the following difference equation

$$
\bar{u}^{p}(m, n)=\bar{a}(m, n)+\sum_{s=m+1}^{\infty} \bar{b}(s, n) \bar{u}^{p}(s, n)+\sum_{s=m+1}^{\infty} \sum_{t=n+1}^{\infty}\left[\bar{F}_{1}(s, t, \bar{u}(s, t))+\sum_{\xi=s}^{\infty} \sum_{\eta=t}^{\infty} \bar{F}_{2}(\xi, \eta, \bar{u}(\xi, \eta))\right],
$$

where $\bar{F}_{1}, \bar{F}_{2}: \Omega \times \mathbb{R} \rightarrow \mathbb{R}$, then

$$
\left|u^{p}(m, n)-\bar{u}^{p}(m, n)\right| \leq(2 \varepsilon)^{\frac{1}{p}} K,
$$


provided that $\widetilde{G}(m, n) \leq K$, where

$$
\begin{array}{r}
\widetilde{G}(m, n)=\left\{\left\{1+\left\{\sum_{s=m+1}^{\infty} \sum_{t=n+1}^{\infty}\left[f_{1}(s, t) J(s, t)+\sum_{\xi=s}^{\infty} \sum_{\eta=t}^{\infty} f_{2}(\xi, \eta) J(\xi, \eta)\right]\right\} \times\right.\right. \\
\left.\left.\prod_{s=m+1}^{\infty}\left\{1+\sum_{t=n+1}^{\infty}\left[\tilde{f}_{1}(s, t)+\sum_{\xi=s}^{\infty} \sum_{\eta=t}^{\infty} \tilde{f}_{2}(\xi, \eta)\right]\right\}\right\} J(m, n)\right\}^{\frac{1}{p}},
\end{array}
$$

and

$$
\tilde{f}_{1}(m, n)=f_{1}(m, n) J(m, n), \tilde{f}_{2}(m, n)=w(m, n) J(m, n), J(m, n)=\prod_{s=m+1}^{\infty}[1+|b(s, n)|] .
$$

The proof for Theorems 3.5-3.6 is similar to Theorems 3.2-3.3, in which Theorem 2.6 is used. Due to the limited space, we omit it here.

\section{Conclusions}

In this paper, some new finite difference inequalities in two independent variables are established, which can be used as a handy tool in the study of boundedness, uniqueness, continuous dependence on initial data of solutions of certain difference equations. The established inequalities generalize some existing results in the literature.

\section{Competing interests}

The authors declare that they have no competing interests.

\section{Authors'contributions}

QF carried out the main part of this article. All authors read and approved the final manuscript.

\section{Acknowledgements}

This work is supported by National Natural Science Foundation of China (Grant No 10571110), Natural Science Foundation of Shandong Province (ZR2009AM011 and ZR2010AZ003) (China) and Specialized Research Fund for the Doctoral Program of Higher Education (20103705110003)(China). The authors thank the referees very much for their careful comments and valuable suggestions on this paper.

\section{Author details}

${ }^{1}$ School of Science, Shandong University of Technology, Zhangzhou Road 12, Zibo, Shandong, 255049, China ²School of Mathematical Sciences, Qufu Normal University, Qufu, Shandong, 273165, China

Received: 28 February 2011 Accepted: 15 July 2011 Published: 15 July 2011

\section{References}

1. Zhao XQ, Zhao QX, Meng FW: On some new nonlinear discrete inequalities and their applications. J Ineq Pure Appl Math 2006, 7:1-9, Article 52

2. Pachpatte BG: Inequalities applicable in the theory of finite differential equations. J Math Anal App/ 1998, 222:438-459.

3. $\mathrm{Ma} \mathrm{QH}: \mathrm{N}$-independent-variable discrete inequalities of Gronwall-Ou-lang type. Ann Differen Equations 2000, 16:813-820.

4. Pachpatte BG: On some new inequalities related to a certain inequality arising in the theory of differential equations. J Math Anal Appl 2000, 251:736-751.

5. Cheung WS, Ma QH, Pečarić J: Some discrete nonlinear inequalities and applications to difference equations. Acta Math Scientia 2008, 28(B):417-430.

6. Deng SF: Nonlinear discrete inequalities with two variables and their applications. Appl Math Comput 2010, 217:2217-2225

7. Cheung WS, Ren JL: Discrete nonlinear inequalities and applications to boundary value problems. J Math Anal Appl 2006, 319:708-724.

8. $\mathrm{Ma} \mathrm{QH}$, Cheung WS: Some new nonlinear difference inequalities and their applications. J Comput Appl Math 2007, 202:339-351. 
9. $\mathrm{Ma} \mathrm{QH}$ : Estimates on some power nonlinear Volterra-Fredholm type discrete inequalities and their applications. J Comput Appl Math 2010, 233:2170-2180.

10. $\mathrm{Ma} \mathrm{QH}$ : Some new nonlinear Volterra-Fredholm-type discrete inequalities and their applications. J Comput Appl Math 2008, 216:451-466.

11. Pang PYH, Agarwal RP: On an integral inequality and discrete analogue. J Math Anal App/ 1995, 194:569-577.

12. Pachpatte BG: On some fundamental integral inequalities and their discrete analogues. J Ineq Pure Appl Math 2001, 2:1-13, Article 15

13. Meng FW, Li WN: On some new nonlinear discrete inequalities and their applications. J Comput Appl Math 2003, 158:407-417.

14. Meng FW, Ji DH: On some new nonlinear discrete inequalities and their applications. J Comput Appl Math 2007, 208:425-433.

15. Jiang FC, Meng FW: Explicit bounds on some new nonlinear integral inequality with delay. J Comput Appl Math 2007, 205:479-486.

doi:10.1186/1687-1847-2011-21

Cite this article as: Feng et al: Some new finite difference inequalities arising in the theory of difference equations. Advances in Difference Equations 2011 2011:21.

\section{Submit your manuscript to a SpringerOpen ${ }^{\circ}$} journal and benefit from:

- Convenient online submission

Rigorous peer review

- Immediate publication on acceptance

- Open access: articles freely available online

- High visibility within the field

- Retaining the copyright to your article

Submit your next manuscript at $\boldsymbol{s p r i n g e r o p e n . c o m ~}$ 